\title{
Correction to: Laminin-derived Ile-Lys-Val-ala-Val: a promising bioactive peptide in neural tissue engineering in traumatic brain injury
}

\author{
Sajad Sahab Negah ${ }^{1,2}$ - Alireza Khooei $^{3}$ • Fariborz Samini $^{4}$ • Ali Gorji ${ }^{1,2,5,6}$
}

Published online: 20 November 2017

(C) Springer-Verlag GmbH Germany, part of Springer Nature 2017

\section{Correction to: Cell and Tissue Research}

https://doi.org/10.1007/s00441-017-2717-6

The published online version contains mistake.

There is only one problem with Table 3 . The references mentioned in this table were wrong in the final proof.

The online version of the original article can be found at https://doi.org/ 10.1007/s00441-017-2717-6

$\triangle$ Ali Gorji

gorjial@uni-muenster.de

1 Department of Neuroscience, Faculty of Medicine, Mashhad University of Medical Sciences, Mashhad, Iran

2 Shefa Neuroscience Research Center, Khatam Alanbia Hospital, Tehran, Iran

3 Department of Pathology, Faculty of Medicine, Mashhad University of Medical Sciences, Mashhad, Iran

4 Department of Neurosurgery, Faculty of Medicine, Mashhad University of Medical Sciences, Mashhad, Iran

5 Department of Neurology and Department of Neurosurgery, Westfälische Wilhelms-Universität Münster, Münster, Germany

6 Epilepsy Research Center, Westfälische Wilhelms-Universität Münster, Robert-Koch-Straße 45, D-4814 Münster, Germany 


\begin{tabular}{|c|c|c|c|c|}
\hline Scaffold Name/Motif & Cell type & Sequences & Results & References \\
\hline IKVAV & BMMSC & $\begin{array}{l}\text { Isoleucyllysyl-valyl-alanyl-v- } \\
\text { aline }\end{array}$ & $\begin{array}{l}\text { Regulate cell growth and } \\
\text { proliferation }\end{array}$ & (Li et al. 2014b) \\
\hline Hydrogels-GIPHs & BMMSC & $\begin{array}{l}\text { Hydrogel-grafted IKVAV poly } \\
\text { (lactide-co-ethylene } \\
\text { oxide-co-fumarate) } \\
\text { (PLEOF) }\end{array}$ & $\begin{array}{l}\text { Provide cell survival and } \\
\text { proliferation }\end{array}$ & (Zhang et al. 2014) \\
\hline $\begin{array}{l}\text { Self-assembled IKVAV } \\
\text { peptide nanofibers }\end{array}$ & $\begin{array}{l}\text { Olfactory } \\
\text { ensheath- } \\
\text { ing cells } \\
\text { (OECs) }\end{array}$ & IKVAV & $\begin{array}{l}\text { Promote cell adhesion and has good } \\
\text { biocompatibility and bioactivity }\end{array}$ & (Xu et al. 2009) \\
\hline $\begin{array}{l}\text { Peptide functionalized } \\
\text { PAMAM dendrimers }\end{array}$ & BMMSC & $\begin{array}{l}\text { RGD, YIGSR or IKVAV } \\
\text { functionalized PAMAM } \\
\text { coated substrate }\end{array}$ & Probably promote cell adhesion & $\begin{array}{l}\text { (Maturavongsadit et al. } \\
\text { 2016) }\end{array}$ \\
\hline IKVAV-PA & $\begin{array}{l}\text { PC12 } \\
\text { NSCs } \\
\text { DRGn }\end{array}$ & $\begin{array}{l}\text { C16H31O-NH-AAAGGGEI- } \\
\text { KVAV-COOH }\end{array}$ & $\begin{array}{l}\text { Promote rapid and stable adherence } \\
\text { of PC } 12 \text { cells } \\
\text { Promote neurite outgrowth } \\
\text { Promote the adhesion of neurons }\end{array}$ & $\begin{array}{l}\text { (Tysseling-Mattiace } \\
\text { et al. 2008; Wu et al. } \\
\text { 2006; Zou et al. } \\
\text { 2009) }\end{array}$ \\
\hline $\begin{array}{l}\text { IKVAV-functionalized } \\
\text { GaN }\end{array}$ & PC12 & $\begin{array}{l}\text { CSRARKQAASIKVAVSAD- } \\
\text { R-NH2 }\end{array}$ & Improved cell adhesion & (Jewett et al. 2012) \\
\hline $\begin{array}{l}\text { Biofunctionalized } \\
\text { peptide-based } \\
\text { hydrogels }\end{array}$ & $\begin{array}{l}\text { Spiral } \\
\text { ganglion } \\
\text { neurons }\end{array}$ & $\begin{array}{l}\text { IKVAV-GGG-RADARADA- } \\
\text { RADARADA-amide } \\
\text { IKVAV-GGG-SIINFEKL }\end{array}$ & Attract neurite outgrowth & (Frick et al. 2016) \\
\hline $\begin{array}{l}\text { IKVAV-containing } \\
\text { peptide }\end{array}$ & NSCs & $\mathrm{C} 16 \mathrm{H} 31 \mathrm{O}-\mathrm{A} 3 \mathrm{G} 4 \mathrm{D} 2 \mathrm{IKVAV}$ & Induce cell growth & (Zheng et al. 2009) \\
\hline E1Y9/E1Y9-IKVAV & PC12 & $\begin{array}{l}\text { Ac-EYEYKYEYKYGGGIK- } \\
\text { VAV-NH2 }\end{array}$ & Promote the neuronal differentiation & (Fukunaga et al. 2016) \\
\hline Collagen-IKVAV & DRG & IKVAV-collagen-RGD & Enhance cell proliferation & $\begin{array}{l}\text { (Hosseinkhani et al. } \\
\text { 2013) }\end{array}$ \\
\hline Silk fibroin-IKVAV & hNSCs & $\begin{array}{l}\text { Silk } \\
\text { fibroin-CSRARKQAASI- } \\
\text { KVAVSADR }\end{array}$ & $\begin{array}{l}\text { Promote the neuronal differentiation, } \\
\text { viability, and proliferation }\end{array}$ & (Sun et al. 2015) \\
\hline Short laminin peptide & hNSCs & $\begin{array}{l}\text { IKVAV: } \\
\text { Ac-Cys-Cys-Arg-Arg-Ile-- } \\
\text { LysVal-Ala-Val-Trp-Leu-C- } \\
\text { ys (CCRRIKVAVWLC) }\end{array}$ & $\begin{array}{l}\text { Increase cell attachment, } \\
\text { proliferation and differentiation } \\
\text { into neurons }\end{array}$ & (Li et al. 2014a) \\
\hline $\begin{array}{l}\text { SIKVAVS-P(HEMA-A- } \\
\text { EMA) }\end{array}$ & MSCs & SIKVAVS-P(HEMA-AEMA & $\begin{array}{l}\text { Enhance attachment and } \\
\text { proliferation }\end{array}$ & (Macková et al. 2016) \\
\hline IKVAVmx & NSCs & $\begin{array}{l}\text { AcN-(RADA)4-GGIKVAV-- } \\
\text { CONH2) }\end{array}$ & $\begin{array}{l}\text { improved cell proliferation and } \\
\text { neuronal differentiation }\end{array}$ & (Zhang et al. 2010) \\
\hline CQIK & $\begin{array}{l}\text { Human } \\
\text { endomet- } \\
\text { rial-- } \\
\text { derived } \\
\text { stromal } \\
\text { cells }\end{array}$ & CQAASIKVAV & Enhance cell viability & (Tavakol et al. 2015) \\
\hline R-GSIK & NSCs & $\begin{array}{l}\text { RADA4-GGSIKVAV } \\
\text { (Ac-RADARADARADA- } \\
\text { RADA-GGS-IKVAVCON- } \\
\text { H2) }\end{array}$ & $\begin{array}{l}\text { Improve cell viability, proliferation, } \\
\text { and neuronal differentiation }\end{array}$ & (Negah et al. 2016) \\
\hline
\end{tabular}

\section{References}

Frick C, Muller M, Wank U, Tropitzsch A, Kramer B, Senn P, RaskAndersen H, Wiesmüller KH, Lowenheim H (2016) Biofunctionalized peptide-based hydrogels provide permissive scaffolds to attract neurite outgrowth from spiral ganglion neurons. Colloids Surf B Biointerfaces 149:105-114
Fukunaga K, Tsutsumi H, Mihara H (2016) Cell differentiation on diskand string-shaped hydrogels fabricated from $\mathrm{Ca} 2+$-responsive selfassembling peptides. Biopolymers 106(4):476-83

Hosseinkhani H, Hiraoka Y, Li C-H, Chen Y-R, Yu D-S, Hong PD, Ou K-L (2013) Engineering three-dimensional collagenIKVAV matrix to mimic neural microenvironment. ACS Chem Neurosci 4(8):1229-1235 
Jewett SA, Makowski MS, Andrews B, Manfra MJ, Ivanisevic A (2012) Gallium nitride is biocompatible and non-toxic before and after functionalization with peptides. Acta Biomater 8(2):728-733

Li X, Liu X, Josey B, Chou CJ, Tan Y, Zhang N, Wen X (2014a) Short laminin peptide for improved neural stem cell growth. Stem Cells Transl Med 3(5):662-670

Li B, Qiu T, Zhang P, Wang X, Yin Y, Li S (2014b) IKVAV regulates ERK1/2 and Akt signalling pathways in BMMSC population growth and proliferation. Cell Prolif 47(2):133-145

Macková H, Plichta Z, Proks V, Kotelnikov I, Kučka J, Hlídková H et al (2016) RGDS-and SIKVAVS-modified Superporous poly (2hydroxyethyl methacrylate) scaffolds for tissue engineering applications. Macromol Biosci 16(11):1621-1631

Maturavongsadit P, Bi X, Gado TA, Nie Y-Z, Wang Q (2016) Adhesive peptides conjugated PAMAM dendrimer as a coating polymeric material enhancing cell responses. Chin Chem Lett 27:1473-1478

Negah SS, Khaksar Z, Aligholi H, Sadeghi SM, Mousavi SMM, Kazemi H, Jahanbazi Jahan-Abad A, Gorji A (2016) Enhancement of neural stem cell survival, proliferation, migration, and differentiation in a novel self-assembly peptide Nanofibber scaffold. Mol Neurobiol 54: 8050-8062

Sun W, Incitti T, Migliaresi C, Quattrone A, Casarosa S, Motta A (2015). Viability and neuronal differentiation of neural stem cells encapsulated in silk fibroin hydrogel functionalized with an IKVAV peptide. J Tissue Eng Regen Med 11(5):1532-1541

Tavakol S, Saber R, Hoveizi E, Tavakol B, Aligholi H, Ai J, Rezayat SM (2015) Self-assembling peptide nanofiber containing long motif of Laminin induces neural differentiation, tubulin polymerization, and neurogenesis: in vitro, ex vivo, and in vivo studies. Mol Neurobiol 53(8):5288-99

Tysseling-Mattiace VM, Sahni V, Niece KL, Birch D, Czeisler C, Fehlings MG et al (2008) Self-assembling nanofibers inhibit glial scar formation and promote axon elongation after spinal cord injury. J Neurosci 28(14):3814-3823

Wu Y, Zheng Q, Du J, Song Y, Wu B, Guo X (2006) Self-assembled IKVAV peptide nanofibers promote adherence of PC12 cells. J Huazhong Univ Sci Technolog Med Sci 26:594-596

Xu H, Shao Z, Wu Y, Deng C, Yu X, Ding F, Zhang B, Xu W (2009) Effects of self-assembled IKVAV peptide nanofibers on olfactory ensheathing cells. Sheng Wu Gong Cheng Xue Bao 25(2):292-298

Zhang ZX, Zheng QX, Wu YC, Hao DJ (2010) Compatibility of neural stem cells with functionalized self-assembling peptide scaffold in vitro. Biotechnol Bioprocess Eng 15(4):545-551

Zhang P, Yin Y, Qiu T, Tao Y, Wang X (2014) Cytocompatibility evaluation of grafted IKVAV PLEOF hydrogels with bone marrow mesenchymal stem cells. Journal of Wuhan University of TechnologyMater Sci Ed 29(4):824-831

Zheng Q, Wu Y, Guo X (2009) Two-dimensional effects of hydrogel selforganized from IKVAV-containing peptides on growth and differentiation of NSCs. Journal of Wuhan University of Technology-Mater Sci Ed 24(2):186-192

Zou Z, Zheng Q, Wu Y, Song Y, Wu B (2009) Growth of rat dorsal root ganglion neurons on a novel self-assembling scaffold containing IKVAV sequence. Mater Sci Eng C 29(7):2099-2103 